\title{
El complejo Myotis nattereri en Iberia: una larga historia
}

\author{
Irene SAliciIni, Carlos IbáÑez, JaVIER JuSte
}

Departamento de Ecología Evolutiva,

Estación Biológica de Doñana (EBD-CSIC)

Correo electrónico del autor: irene@ebd.csic.es

DOI: http://dx.doi.org/10.14709/BarbJ.5.1.2012.01

English title: The Myotis nattereri complex in Iberia: a long history

\begin{abstract}
More than a century after the description of Myotis escalerai by Ángel Cabrera, modern genetic tools now shed new light on the enigma of the Myotis nattereri species complex. Using different genetic markers (mitochondrial and nuclear) and different approaches in the analysis, we reveal the presence of four different clades corresponding to phylogenetic species. Two new species of Myotis besides M. nattereri and M. escalerai are identified for the Western Palearctic. Nevertheless, taxonomic and morphologic descriptions are necessary if these new species are to be officially recognized. Two of these four species are present in the Iberian Peninsula; their taxonomic situation, distribution and the presence of diagnostic characters are discussed.
\end{abstract}

Keywords: Western Palearctic, phylogeny, species delimitation, Myotis nattereri, Myotis escalerai.

Resumen: A más de un siglo de la descripción de Myotis escalerai por Ángel Cabrera, las herramientas genéticas permiten ahora arrojar nueva luz sobre el enigma del complejo especifico Myotis nattereri. La utilización de diferentes marcadores moleculares (mitocondriales y nucleares) y la combinación de diferentes métodos de análisis, demuestra la presencia de cuatro clados que corresponden a especies filogenéticas. Además de M.nattereri y M. escalerai, dos especies nuevas para la ciencia son reconocidas en el Paleártico Occidental. Su descripción taxonómica y descripción morfológica es fundamental para que puedan ser reconocidas oficialmente como especies. En la Península Ibérica están presentes dos de los cuatro taxones, su situación taxonómica, distribución y los caracteres para reconocerlos son tratados en este artículo.

Palabras clave: Paleártico Occidental, filogenia, delimitación de especies, Myotis nattereri, Myotis escalerai.

\section{INTRODUCCIÓN}

El primero en notar algo raro fue Ángel Cabrera. En su revisión de los quirópteros de España del 1904 describe una nueva especie: Myotis escalerai, muy parecido a $M$. nattereri, pero con alguna diferencia en el color del pelaje y en la inserción del ala en el tobillo. El misterio empieza a los pocos años. Él mismo, solo diez años después, declara estar convencido que "Escalerai es simplemente un sinónimo de Nattereri, sin que pueda separársele ni aun como forma local" por haber visto ejemplares en España sin los caracteres de $M$. escalerai, y por el contrario $M$. nattereri del extranjero que presentaban esos caracteres que se suponían únicos de los escalerai ibéricos.

Más de un siglo después de la descripción original de Myotis escalerai por Cabrera, la genética podía mostrar lo que la morfología parecía tener escondido. Los primeros análisis genéticos confirmaron la presencia en Iberia de un grupo de nattereri muy diferente de sus familiares Centro-Norte Europeos, probablemente correspondiente a los escalerai de Cabrera.

La genética reveló además un segundo clado en el Norte de España, desconocido hasta el momento. Este segundo grupo se diferenciaba del $M$. nattereri "clásico" y también del supuesto $M$. escalerai. Como a todos los desconocidos le fue dado un nombre ficticio, de utilidad: Myotis spA (Ibáñez et al., 2006). Para mayor complicación, un tercer nuevo linaje aparecía en Marruecos (Myotis spB) (Ibáñez et al. 2006; García-Mudarra et al. 2009).

Desde entonces hemos acumulado muchos dados, analizado muchas muestras, gracias a una intensa colaboración y a un sincero interés en aclarar este misterio por parte de 
muchos miembros de la SECEMU. Y los datos han venido confirmando cada vez más esos primeros resultados y aportando importantes informaciones sobre la distribución de los linajes. ¿Que faltaba para poder decir que son especies diferentes? ¿Porqué hacían falta más y más análisis genéticos?

\section{LA GENÉTICA: UNA PODEROSA HERRAMIENTA EN CONTINUO CAMBIO}

La taxonomía tradicional, basada en caracteres morfológicos, puede resultar complicada por fenómenos evolutivos como la convergencia o la presencia de caracteres plesiomórficos (caracteres ancestrales que se mantienen a lo largo de la evolución), fenómenos que parecen ser especialmente comunes en el grupo de los quirópteros (Burland \& Worthington Wilmer 2001; Mayer \& Helversen 2001). La utilización de técnicas moleculares constituye en estos casos una preciosa herramienta para reconocer los límites de las especies y ha supuesto en efecto una importante mejora en nuestra capacidad de detectar especies imposibles de reconocer con caracteres morfológicos, o sea las denominadas especies crípticas. Muchos son los ejemplos en el grupo de los microquirópteros, como en el caso M. mystacinus/ alcathoe (Helversen et al. 2001) y en los géneros Plecotus (Kiefer et al. 2002; Spitzenberger et al. 2006) o Miniopterus (Appleton et al. 2004; Goodman et al. 2009).

Indudablemente la genética es una herramienta muy poderosa. Pero al fin solo es eso, una herramienta; un instrumento que podemos utilizar para ver cosas que sería imposible apreciar tan solo con estos cinco sentidos tan limitados que tenemos, como un microscopio o un detector de ultrasonidos. Pero ningún instrumento nos dice que es lo que estamos viendo o escuchando, solo sirven de conexión entre nosotros y el objeto de nuestro interés.

Si queremos utilizar esta herramienta no solo para ver algo, sino más bien para reconocer "cosas" nuevas, para describir una realidad que no conocíamos, se hace aun más difícil poner a todos de acuerdo, sobre todo cuando las técnicas y las teorías se van renovando continuamente. La genética es seguramente uno de los campos de las biología que más rápidamente ha evolucionado desde sus primeras aplicaciones prácticas, aun muy recientes comparadas con la larga historia del estudio de la biología. Nuevas teorías, nuevos métodos analíticos, nuevos instrumentos, significan nuevas potencialidades, pero también diferentes enfoques y muchos debates.

\section{DELIMITACIÓN GENÉTICA DE NUEVAS ESPECIES}

Lo que pretendemos hacer cuando utilizamos la genética para detectar los límites de una especie, es acercarnos lo más posible a la definición de especie: detectar si efectivamente el flujo genético entre dos grupos es nulo o tan limitado que los dos grupos representan dos líneas evolutivas independientes. Sin embargo, para evitar un aumento artificial en el número de especies reconocidas (Isaac et al. 2004), la utilización del concepto filogenético de especie (basado en el reconocimiento de caracteres evolutivos derivados que sean únicos y comunes a los miembro del clado) debe aplicarse con cuidado y debe concentrarse el máximo énfasis en la fiabilidad y mejora de los métodos utilizados.
Hasta hace pocos años la mayoría de los análisis filogenéticos se basaban simplemente en el ADN mitocondrial. Los avances teóricos han dejado claro que el ADN mitocondrial solo cuenta una parte de la historia evolutiva de un clado, que no siempre corresponde a la historia completa (Ballard \& Whitlock 2004). La herencia materna del ADN mitocondrial hace que solo la historia de las hembras se refleje en este material genético, y esto puede ser un problema en especies con profundas diferencias de comportamiento entre los dos sexos (por ejemplo en caso de fuerte filopatría de las hembras y dispersión de los machos). Por otro lado, debido a la ausencia de recombinación en la transmisión del ADN mitocondrial, este se comporta como una unidad única. Por lo tanto el árbol genético que se obtiene es equivalente a un "gene-tree" y puede no corresponder al árbol filogenético completo de toda la especie ("species-tree") (ver por ejemplo la revisión de Degnan \& Rosenberg 2009)

Por estas razones, haber notado algo diferente en la morfología y haber encontrado unos linajes mitocondriales que indicaban una profunda separación genética en este marcador, todavía no eran pruebas suficientes para poder decir que efectivamente lo que habíamos encontrado correspondían a nuevas, diferentes especies. Para disipar las dudas sobre la identidad taxonómica todavía hacía falta añadir a los análisis el ADN nuclear.

\section{Metodología y ReSUltados}

Con el objetivo de aclarar los límites de las especies presentes en el complejo específico del murciélago M. nattereri s.l. y determinar las relaciones filogenéticas entre ellas, hemos analizado 32 muestras de este taxón procedentes de diferentes regiones del Paleártico Occidental, incluyendo varios países de Europa además de Marruecos.

Este estudio, publicado recientemente (Salicini et al. 2011), da un primer paso hacia la resolución del rompecabezas del grupo Myotis nattereri. Aquí damos un resumen de los resultados obtenidos, centrándonos en las consecuencias taxonómicas, y en la conservación, estudio y manejo de la quiropterofauna Ibérica.

Se han secuenciado dos marcadores mitocondriales (Cytb y ND1) y 6 intrones nucleares (SLC38A7-8, ABHD11-5, ACOX2-3, ACPT-4, COPS7A-4 y ROGDI- 7; Igea et al. 2010), con un total de 3535 pares de bases. Dos ejemplares de Myotis schaubi de Irán han sido añadidos al estudio para analizar su posición filogenética en relación al grupo nattereri.

Los intrones son regiones del ADN que no codifican proteínas, por esta razón su tasa de mutación (o sea la velocidad con la que van acumulando los pequeños cambios que nos sirven para reconstruir la historia evolutiva de una secuencia) es mayor que la tasas de otras regiones del ADN nuclear, generalmente muy conservado y por esto poco informativo a nuestro nivel. Además, no codificando en la producción de proteínas, su evolución no está directamente afectada por la selección natural, siendo entonces testigos "neutrales" de la historia evolutiva (Creer 2005).

Los datos genéticos han sido analizados con diferentes metodologías y en diferentes combinaciones para poder comparar y corroborar los resultados. Ninguno de los 
intrones por si solo es bastante variable para ser informativo. Sin embargo al reconstruir el árbol filogenético con los seis marcadores nucleares juntos, la información sobre la evolución de los taxa estudiados es evidente y resulta ampliamente coincidente con el resultado del árbol filogenético mitocondrial (Fig.1).
Por el momento su distribución parece típicamente ibérica, aunque ejemplares de esta especie han sido capturados en la vertiente norte de los Pirineos Franceses (Evin et al. 2009).

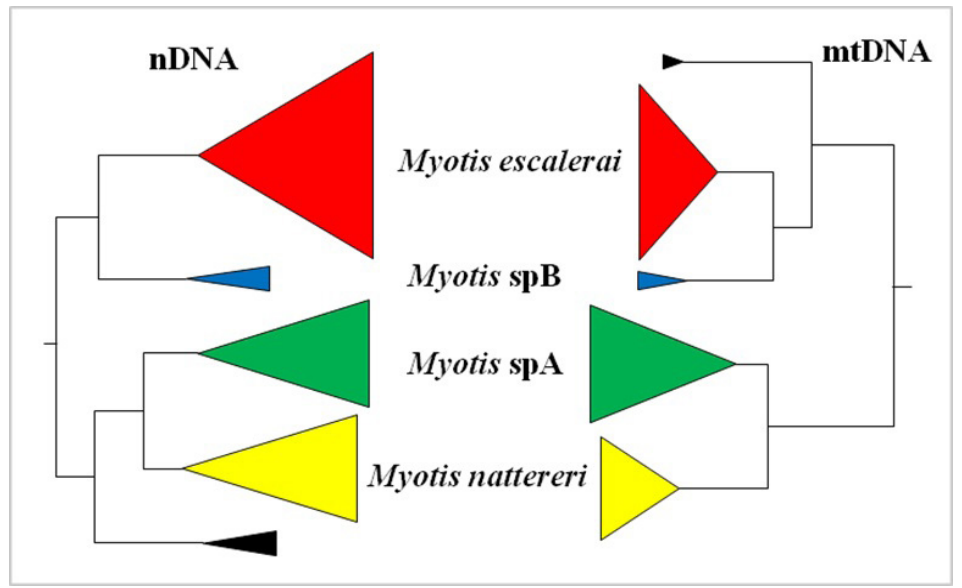

Fig. 1. Árboles filogenéticos basados en seis marcadores nucleares (izquierda) y dos marcadores mitocondriales (derecha) concatenados. Adaptado de Salicini et al. 2011

En ambas reconstrucciones filogenéticas se evidencian cuatro clados bien diferenciados dentro de Myotis nattereri s.l., además de $M$. schaubi.

Los resultados obtenidos con el ADN nuclear corroboran que la robusta estructura encontrada previamente en el ADN mitocondrial no depende exclusivamente de una fuerte filopatría de las hembras. Los cuatros taxones encontrados han estado en efecto separados evolutivamente tanto tiempo que esta división está presente no solo en el ADN mitocondrial (de evolución más rápida) sino también en el ADN nuclear. Con nuestros análisis no podemos excluir que haya habido sucesivo flujo genético entre ellos, pero si hay o ha habido 'inter-breeding', éste ha sido lo suficientemente limitado para que la señal de la profunda división no se haya visto afectada.

\section{DisCuSIÓN Y CONCLUSIONES}

Podemos entonces concluir que los cuatro linajes encontrados corresponden a especies según el concepto filogenético de especie.

Para dos de las cuatro especies existe ya una descripción taxonómica.

Myotis nattereri s.str. (en adelante simplemente $M$. nattereri) fue descrito en 1817 por Kuhl con ejemplares procedentes de Alemania Central. Esta especie está ampliamente distribuida en Europa Central, Septentrional y Oriental; sin embargo hasta el momento no ha sido encontrada ni en la Península Italiana, ni en Iberia.

La descripción de Myotis escalerai hecha por Cabrera en 1904 es válida, ya que por los menos uno de los ejemplares estudiados por el zoólogo procedía de un área (Valencia) donde solo se encuentra este linaje. El nombre de esta especie puede entonces ser rescatado, tras haber sido considerado sinónimo de $M$. nattereri por muchos años. Sus características morfológicas, ecológicas y su distribución necesitan ser estudiadas a la luz de las novedades genéticas.
Diferente es la situación de los dos nuevos clados:

Myotis spA es el segundo clado encontrado en la Península Iberica y el único presente en la Península Italiana. Genéticamente es especie hermana de $M$. nattereri.

Myotis spB es el único nattereri encontrado en la región del Magreb. Genéticamente y morfológicamente es cercano a $M$. escalerai. Muy pocas son las informaciones existentes sobre la distribución y el estado real de conservación del clado en esta región, donde se considera una especie rara (Benda et al. 2004).

\section{SigUIENTES PASOS TAXONÓMICOS}

Hemos demostrado la naturaleza genética de estos dos linajes, pero todavía oficialmente no existen como especies. Como un niño recién nacido no existe oficialmente como "persona jurídica" hasta que no se cumplan una serie de trámites para su inscripción en el registro civil, para una nueva especie hay que proceder de acuerdo a las reglas del Código Internacional de Nomenclatura Zoológica para que su existencia y su descripción se consideren válidas y puedan ser aceptadas por toda la comunidad científica.

Hasta que no se haga una descripción de las nuevas especies según estas reglas, no podremos realmente tratar estos clados como especies diferentes, ni utilizar nombres científicos, cuya elección no puede adelantarse a esa descripción taxonómica "clásica".

Con el fin de realizar dicha descripción taxonómica y aclarar formalmente la división en diferentes especies de este clado tan complejo, en los últimos meses hemos estado recolectando muchos datos morfológicos cuantitativos (medidas morfométricas) y cualitativos (fotografías de caracteres fenotípicos) tanto en ejemplares de museos como en animales capturados vivos. Los análisis estadísticos de estos datos todavía no han empezado, sin embargo algunas indicaciones ya aparecen en los caracteres cualitativos, aunque siguen siendo especies crípticas muy difíciles de distinguir con caracteres fenotípicos. 
Especialmente compleja es la distinción entre los elementos dentro de las dos parejas de especies M.nattereri/M.spA y M.escalerai/M.spB, para los cuales todavía no se han encontrado caracteres distintivos.

Al contrario, se confirman algunos caracteres que permiten distinguir entre los dos grupos (M.nattereri/M.spA versus M.escalerai/M.spB). El carácter probablemente más evidente, y ampliamente comprobado, es la diferencia en las franja de pelos del margen del uropatagio (Fig.2). La franja ventral interna en $M$. escalerai y M.spB presenta pelos más largos, más densos y en dirección al cuerpo del animal (Fig.2a).

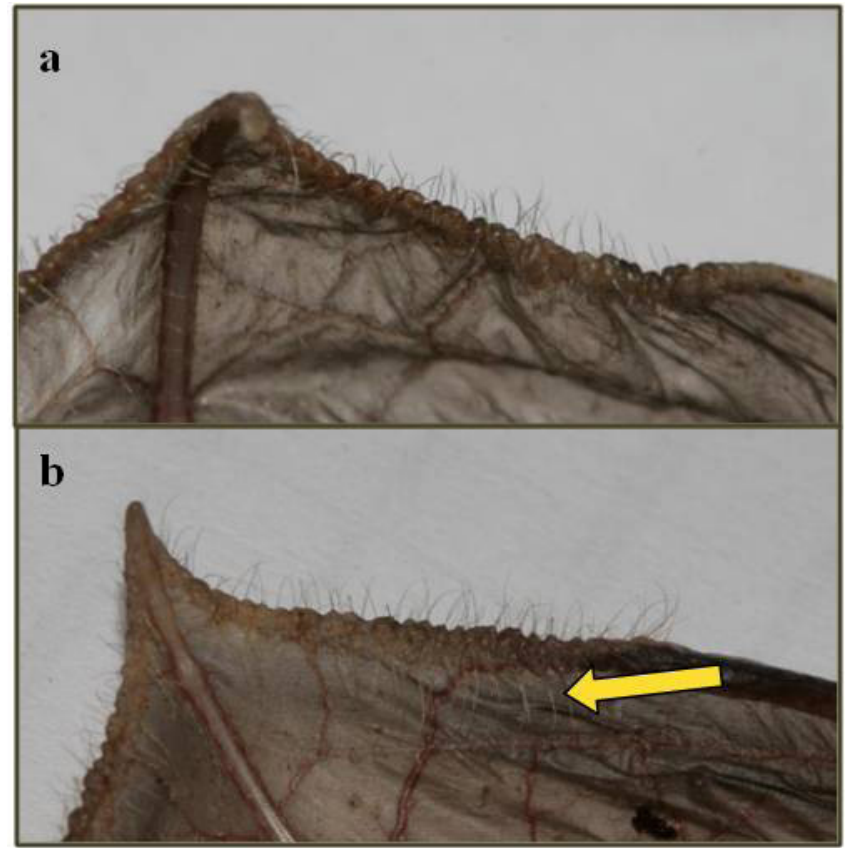

Fig. 2. Detalle del borde del uropatagio de $M \cdot \operatorname{spA}$ (a) y $M$. escalerai (b). La flecha indica los pelos ventrales característicos de M. escalerai.

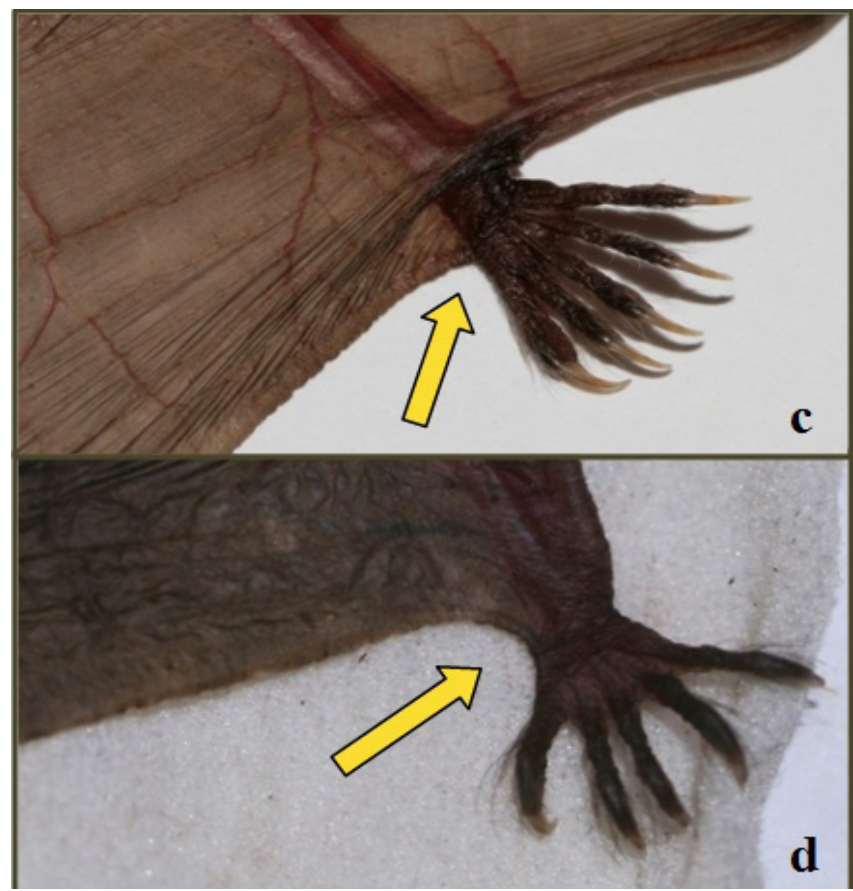

Fig. 3. Detalle de la inserción de la ala en el tobillo de $M$.spA (c) y M. escalerai (d). Las flechas indican el diferente ángulo en la inserción.
La inserción del ala en las patas parece también presentar una forma diferente en los dos grupos (fig.3), aunque su consistencia no ha sido comprobada lo suficiente en las cuatro especies.

\section{SITUACIÓN EN IBERIA}

Como hemos dicho, en la Península Ibérica aparecen ejemplares de dos de los cuatros clados encontrados en el grupo nattereri. Myotis escalerai está presente en toda la península, estando el segundo taxón limitado a áreas septentrionales hasta el norte de Extremadura por occidente y hasta Cataluña en la costa oriental. También parece evidente una división altitudinal entre las dos especies, siendo $M$. escalerai más frecuente a bajas cotas, mientras que Myotis spA resulta presente a mayores altitudes. Muy escasos son los sitios de simpatria donde las dos especies pueden coincidir espacialmente, por los menos en temporada de "swarming".

Estas dos especies son bien distintas genéticamente y, aunque el estudio de los caracteres morfológicos está en su comienzo, parece confirmarse la consistencia de algunos caracteres útiles para distinguirlos.

La observación de los caracteres descritos en el precedente apartado (franja de pelo del uropatagio e inserción de la ala en la pata) permite asignar con relativa seguridad los ejemplares a una u otra especie. Aun así el análisis genético es todavía la única forma de confirmar la especie con certeza. Conocer las especies existentes no es solamente una mera curiosidad taxonómica, al contrario es el primer paso fundamental para cualquier estudio posterior de ecología y la base para la conservación de la propia biodiversidad (Agapow et al. 2005; Sattler et al. 2007). El reconocimiento de nuevas especies en el Paleártico Occidental demuestra cuanto queda todavía por descubrir en la biodiversidad, incluso en un área tan ampliamente estudiada como es la cuenca del Mediterráneo. La distribución limitada de los nuevos clados encontrados reclama más informaciones sobre la situación real de conservación de cada uno de ellos. Los dos clados presentes en Iberia se encuentran limitados a regiones reducidas y bajo fuerte presión antropogénica. Esta situación sugiere la necesitad de reconsiderar tanto el estado de estas especies como los planes para sus conservación.

\section{Algunas indicaciones prácticas}

A la espera de las descripciones taxonómicas oficiales de los dos nuevos clados, no podemos, como ya hemos dicho, dar un nombre a la nueva especie presente en España. La forma que nos parece más correcta para hablar de esta especie, por ejemplo a la hora de publicar guías de murciélagos o de redactar listas de especies, es indicarla como Myotis $c f$. nattereri (seguramente más oportuno en publicaciones que Myotis $\operatorname{sp} A$ ), especificando que los resultados de recientes estudios genético han evidenciado que se trata de un linaje muy diferente (Salicini et al. 2011) y que probablemente se trate de una nueva especie en espera de descripción.

En el caso de M. escalerai ya podemos utilizar este nombre, aunque su descripción todavía necesita ser actualizada y rescatada. Los caracteres morfológicos aquí descritos y las fotografías presentadas deben utilizarse con prudencia, ya que todavía no tenemos los resultados finales de estos análisis. 


\section{Agradecimientos}

Todo este trabajo ha sido posible gracias a la preciosa colaboración de muchos colegas, entre los cuales queremos destacar: J.L. García-Mudarra, A. Fijo, J.A. Garrido, J. Quetglas, I. Blázquez de Paz, J. Nogueras, E. Migens, P.T. Agirre-Mendi, J.T. Alcalde, D. Almenar, M.A. Monsalve, T. Castelló, C. Flaquer, X. Puig, A. López-Baucells, D. García, G. Schreur, D. Trujillo, J. Aihartza, I. Garin, M. Napal, R. Hermida, F. Lamas, X. Parda Vila, F. Carro, O. de Paz, G. Pérez- Suárez, J. de Lucas, F. González, F. Lisón, P. García, H. Rebelo, P. Barros.

\section{REFERENCIAS}

Agapow, P.M., 2005. Species: demarcation and diversity. In: Phylogeny and Conservation (eds PurvisA, GittlemanJL, BrooksT), Cambridge University Press, $57-75$.

Appleton, B.R., McKenzie, J.A., Christidis, L., 2004. Molecular systematics and biogeography of the bentwing bat complex Miniopterus schreibersii (Kuhl, 1817) (Chiroptera: Vespertilionidae). Molecular Phylogenetics and Evolution, 31, 431-439.

Ballard, J.W.O., Whitlock, M.C., 2004. The incomplete natural history of mitochondria. Molecular Ecology, $13,729-744$

Benda, P., Ruedi, M., Aulagnier, S., 2004. New data on the distribution of bats (Chiroptera) in Morocco. Vespertilio, 8, 13-44.

Burland, T.M., Worthington Wilmer, J., 2001. Seeing in the dark: molecular approaches to the study of bat populations. Biological Reviews of the Cambridge Philosophical Society, 76, 389-409.

CABrera, A., 1904. Ensayo monográfico sobre los quirópteros de España. Memorias de la Real Sociedad Española de Historia Natural 2, 5, 249-287.

CAbrera, A., 1914. Fauna iberica. Mamíferos. Museo Nacional de Ciencias Naturales, Madrid.

Creer, S., Malhotra, A., Thorpe, R.S., Pook, C.E., 2005. Targeting optimal introns for phylogenetic analyses in non-model taxa: experimental results in Asian pitvipers. Cladistics, 21, 390-395. DOI: https://dx.doi. org/10.1111/j.1096-0031.2005.00072.x

Degnan, J.H., Rosenberg, N.A., 2009. Gene tree discordance, phylogenetic inference and the multispecies coalescent. Trends in Ecology \& Evolution, 24,332-340. DOI: https://dx.doi.org/10.1016/j.tree.2009.01.009

Evin, A., Lecoq, V., Durand, M.O., Tillon, L., Pons, J.M., 2009. A new species for the French bat list: Myotis escalerai (Chiroptera: Vespertilionidae). Mammalia, 73, 142-144. DOI: https://dx.doi.org/10.1515/ MAMM.2009.030
García-Mudarra, J.L., IbáÑez, C., Juste, J., 2009. The Straits of Gibraltar: barrier or bridge to Ibero-Moroccan bat diversity? Biological Journal of the Linnean Society, 96, 434-450. DOI: https://dx.doi.org/10.1111/j.1095$\underline{8312.2008 .01128 . x}$

Goodman, S.M., Maminirina, C.P., Weyeneth, N., Bradman, H.M., Christidis, L., Ruedi, M., Appleton, B., 2009. The use of molecular and morphological characters to resolve the taxonomic identity of cryptic species: the case of Miniopterus manavi (Chiroptera, Miniopteridae). Zoologica Scripta, 38, 339-363. DOI: https://dx.doi.org/10.1111/j.1095-8312.2008.01128.x

Helversen, O., Heller, K.-G., Mayer, F., Nemeth, A., Volleth, M., Gomвкото, P., 2001. Cryptic mammalian species: a new species of whiskered bat (Myotis alcathoe n. sp.) in Europe. Naturwissenschaften, 88, 217-223. DOI: https://dx.doi.org/10.1007/s001140100225

IbáÑez, C., García-Mudarra, J.L., Ruedi, M., Stadelmann, B., Juste, J., 2006. The Iberian contribution to cryptic diversity in European bats. Acta Chiropterologica, 8, 277-297. DOI: http://dx.doi.org/10.3161/17335329(2006)8[277:TICTCD]2.0.CO;2

IgeA, J., Juste, J., CASTRESAna, J., 2010. Novel intron markers to study the phylogeny of closely related mammalian species. BMC Evolutionary Biology, 10, 369. DOI: https://dx.doi.org/10.1186/1471-2148-10-369

IsaAc, N.J.B., Mallet, J., Mace, G.M., 2004. Taxonomic inflation: its influence on macroecology and conservation. Trends in Ecology \& Evolution, 19, 464 469. DOI: http://dx.doi.org/10.1016/j.tree.2004.06.004

Kiefer, A., Veith, M., 2002. A new species of long-eared bat from Europe (Chiroptera: Vespertilionidae). Myotis, 39, 5-16. DOI: http://dx.doi.org/10.3161/001.004.0202

Mayer, F., von Helversen, O., 2001. Cryptic diversity in European bats. Proceedings of the Royal Society B, 268, 1825-1832. DOI: https://dx.doi.org/10.1098/ rspb.2001.1744

SALICINI, I., IBÁÑEZ, C., Juste, J., 2011. Multilocus phylogeny and species delimitation within the Natterer's bat species complex in the Western Palearctic. Molecular Phylogenetics and Evolution, 61, 888- 898. DOI: https://dx.doi.org/10.1016/i.ympev.2011.08.010

Sattler, T., Bontadina, F., Hirzel, A.H., Arlettaz, R., 2007. Ecological niche modelling of two cryptic bat species calls for a reassessment of their conservation status. Journal of Applied Ecology, 44(6), 1188-1199.

Spitzenberger, F., Strelkov, P.,Winkler, H., Haring, E., 2006. A preliminary revision of the genus Plecotus (Chiroptera, Vespertilionidae) based on genetic and morphological results. Zoologica Scripta, 35, 187-230. 